\title{
Las niñas también quieren jugar: la participación conjunta de niños y niñas en actividades físicas no organizadas en el contexto escolar
}

\author{
Pedro Gil-Madrona* \\ Javier Cachón-Zagalaz** \\ Arturo Diaz-Suarez ${ }^{* * *}$ \\ Pedro Valdivia-Moral ${ }^{* * * *}$ \\ Maria Luisa Zagalaz-Sánchez $z^{* * * *}$
}

\begin{abstract}
Resumen: Presentamos un estudio empírico descriptivo realizado con 250 participantes de 6을 Educación Primaria (EP), 130 niñas y 120 niños de cuatro centros escolares españoles con distintas características, aplicándose una encuesta de 10 ítems para comprender las barreras que encuentran las chicas para la realización de actividad física (AF) y deporte, en su tiempo de ocio escolar. Los resultados indican que existen diferencias de género a la hora de practicar juegos y deportes, aunque se está produciendo un cambio de conducta que hace que chicos y chicas se agrupen con mayor frecuencia para la realización de AF y deportes.

Palabras clave: Actividad motora. Deportes. Actividades recreativas. Identidad de género. Educación primaria y secundaria.
\end{abstract}

\footnotetext{
'Facultad de Educación, Campus Universitario de Albacete. Universidad de Castilla La Mancha. España. E-mail: pedro.gil@uclm.es

"Facultad de Humanidades y CC de la Educación. Campus de Las Lagunillas. Universidad de Jaén. España. E-mail: jcachon@ujaen.es

"'Facultad de CC del Deporte. Campus de San Javier. Universidad de Murcia. España. E-mail: ardiaz@um.es

*.*.Departamento de Educación Física, Música y Artes Plásticas. Facultad de Educación. Universidad de Huelva. E-mail: pedro.valdivia@dempc.uhu.es

....*Facultad de Humanidades y CC de la Educación. Campus de Las Lagunillas. Universidad de Jaén.E-mail: Izagalaz@ujaen.es
} 


\section{ArtigosOrignais}

\section{INTRODUCCIÓN}

El tema de este trabajo no es cuestión baladí aunque vivamos en el siglo XXI, muy al contrario, la discriminación por género se sigue produciendo en los centros educativos, dentro y fuera de las clases regladas, por eso acometemos un breve e importante estudio, sobre cómo se desenvuelven niños y niñas de $6^{\circ}$ curso de EP en el colegio durante la realización de AF y deporte.

La idea surge en base al proyecto INCLUD-ED que analiza las estrategias educativas que contribuyen a superar las desigualdades y a fomentar la cohesión social, prestando especial atención a grupos vulnerables o desfavorecidos entre los que se encuentran las mujeres, con la intención de diseñar nuevas políticas que permitan alcanzar los objetivos de la Estrategia Europea 2020.

Antes de los 11 o 12 años a los escolares les gusta tomar parte en las clases de EF y en actividades relacionadas como juegos y deportes (GIL-MADRONA; CONTRERAS-JORDÁN, 2003). Sin embargo la AF disminuye a medida que avanza su edad, lo que se agudiza en el caso de las niñas (BIDDLE; GORELY; STENSEL, 2004).

En las últimas tres décadas, ha disminuido el tiempo que las niñas dedican a hacer AF (DWYER et al., 2006), pero los chicos han demostrado repetidamente mayor tasa de participación (EISNMANN; KATZMARYSK; TREMBLAY, 2004; JANSSEN, 2004). Una de las explicaciones de la escasa participación y del abandono deportivo proviene de la socialización del rol de género, ya que las presiones sociales y culturales siguen inculcando a los jóvenes actividades "más adecuadas a su sexo" (HAMZEH, 2007).

El problema de la participación de la mujer en la AF ha sido abordado históricamente desde diferentes perspectivas, afirmando sobre ello Flintoff y Scraton (2001) que la EF contribuye a las relaciones de género y potencia la adquisición de poder de las mujeres. A lo que Valdivia-Moral, López-López, Lara-Sánchez y ZagalazSánchez (2012) añaden que la coeducación en EF, trabajada en las 
etapas de formación en los contextos escolares, ayuda a tener unas relaciones normalizadas entre chicos y chicas.

En la EF actual predominan las ideas sobre salud y rendimiento deportivo, de forma que en muchas ocasiones queda patente el dominio masculino por razones fisiológicas (VALDIVIA-MORAL et al., 2012). Ello contracta con la afirmación de que la inferioridad de las mujeres en habilidades deportivas está asociada a un modelo en el que el aprendizaje no contribuye a la mejora (WOODHILL; SAMMUELS, 2004), donde las chicas tenían miedo a la EF por la dificultad de las tareas y excesiva presión.

Hay estudios sobre la participación de los niños en actividades organizadas (fútbol, hockey...), pero no incluyen acciones informales como jugar en el recreo, en la calle o en los parques antes o después del colegio que son parte importante de la AF (RIDDOCH et al., 2004). Respecto al género femenino, según el Instituto de la Mujer ([IM], 2005) sus actividades preferidas son la natación, el aerobic y la gimnasia de mantenimiento. No obstante, debemos ser conscientes de que no hay estudios en nuestro país que aborden las actividades no organizadas, de ahí la importancia del estudio que abordamos.

Las actividades no organizadas pueden reflejar mejor los hábitos de AF en los escolares porque dependen de su propia motivación. En estos casos, niños y niñas están más predispuestos a seleccionarlas, lo que puede significar una mayor participación. De ahí que, la actividad no organizada sea el centro de esta investigación, ya que para cambiar la AF obligatoria, se debe tomar la opinión de los expertos y la del alumnado para responder a sus inquietudes (CERVELLÓ; JIMENEZ; DEL-VILLAR; RAMOS; SANTOSROSA, 2004; HAMZEH, 2007), quienes destacan que un clima motivacional predice la percepción de igualdad de trato en EF escolar.

Con respecto al profesorado, Zagalaz et al. (2008), tratan de vincular la teoría con la práctica educativa de los futuros maestros para averiguar la valoración que el alumnado de EP otorga a la EF escolar. Concluyen que dicho alumnado valora más positivamente la EF que el resto de las asignaturas del currículum oficial de la etapa, 
por su motivación, interés y disfrute de los contenidos, especialmente juegos y deportes. Estos aspectos influyen en las relaciones que se dan entre el alumnado, tal y como indica Jiménez et al. (2010).

Para saber la necesidad de AF de las chicas observaremos cómo se interesan en la participación (AZZARITO; SOLMON, 2006), tratando de conseguir que formen parte de los procesos de diseño de cualquier forma de intervención (WILSON et al., 2005). De ahí que, como señalan Oliver, Hamzeh y McCaughtry (2009) la investigación deba analizar "lo que es" y "cómo debería de ser" para conseguir "lo que podría ser" y la voluntad de llegar a un cambio progresivo en las prácticas en la EF escolar y extrapolarlo así al tiempo extraescolar y los hábitos de los escolares.

\section{Objetivo}

Analizar la influencia de las barreras existentes por discriminación de género en la práctica de AF y deportes para las chicas de $6^{\circ}$ de EP en los periodos de ocio escolar.

\section{Material y mÉtodo}

Han participado 250 escolares de $6^{\circ}$ de EP, de 4 colegios, situados en 4 localidades de la provincia de Albacete (Castilla-La Mancha), 130 niñas y 120 niños. El nivel escolar es el correspondiente al $6^{\circ}$ curso de ensino fundamental.

Colegio A. 82 sujetos (42 chicas y 40 chicos). La tasa de inmigración o de niños con riesgo de exclusión es nula. Colegio $B$. 50 alumnos (27 chicas y 23 chicos). Niños de familias trabajadoras del sector servicios. Población inmigrante del 9\%. Colegio C. 68 alumnos (35 chicas y 33 chicos). Familias de clase media. El 10\% pertenece a minorías étnicas y el $15 \%$ son extranjeros (rumanos, colombianos, ucranianos, moldavos, rusos, bolivianos, ecuatorianos y peruanos). Colegio D. 50 sujetos (26 chicas y 24 chicos). Proceden de contextos diversos, etnia gitana, origen sudamericano y magrebí. 
El instrumento de recogida de información ha sido un cuestionario de 10 ítems, 3 de tipo Likert (desde N: Nunca; RV: Rara vez; AV: A veces; AM: A menudo; hasta S: Siempre) y 7 con carácter dicotómico (Si/No). Los enunciados de las cuestiones han sido elaborados cuidadosamente para adecuarse a la comprensión de los participantes teniendo en cuenta que se dirige a ambos sexos. Para ello, se llevó a cabo el proceso seguido por Valdivia-Moral, Molero, Campoy y Zagalaz-Sánchez (in press) ${ }^{1}$ para obtener la fiabilidad y validez necesarias teniendo en cuenta el género en EF. Asimismo, para elaborar dichas cuestiones se consideraron los instrumentos elaborados por Castillo, Martínez-López y Zagalaz (2010), Cervelló et al. (2004) y Valdivia-Moral et al. (in press).

Para la validación el cuestionario se envió a jueces expertos, todos investigadores en el campo de la didáctica y la $\mathrm{AF}$ y el deporte, lo que permitió conseguir la validación de contenido tal y como indican Ortega, Calderón, Palao y Puigcerver (2008). Además se realizó una prueba piloto para analizar la validez de comprensión del profesorado (ORTEGA et al., 2008; VALDIVIA-MORAL et al., in press). Tal y como justifican estos autores, se destaca que en el proceso de selección de juezas y jueces expertos se escogieron aquellas personas que cuentan con una dilatada experiencia tanto en el diseño y validación de cuestionarios como en la investigación sobre actividad física y género.

El procedimiento se inicia con la aprobación de los padres o tutores y el permiso de los centros educativos. El cuestionario fue cumplimentado en la clase de EF, en un tiempo aproximado de 15 min., en presencia de un investigador que informó de la voluntariedad para participar y del anonimato de los participantes, además de resolver las dudas de los escolares.

El análisis de los resultados se realizó con los programas informáticos Excel y SPSS (versión 19). Se realizó un análisis de frecuencias que consistió en la descripción de los centros y la

IVALDIVIA-MORAL, P; MOLERO, D.; CAMPOY, T.J. Y ZAGALAZ, M.L. Pensamiento coeducativo del profesorado de EF: Propiedades psicométricas de una escala. Revista Internacional de Medicina y Ciencias de la Actividad Física y del Deporte, in press. 
comparación cualitativa de las puntuaciones obtenidas, expresadas en porcentajes de respuesta en función de la variable género.

Para llevar a cabo este estudio fue necesario acogerse a las orientaciones de los comités de ética de las Universidades de Castilla la Mancha, Jaén, Murcia y Cádiz. No obstante, al no tratarse con parámetros biológicos no fue necesario solicitar el registro de la investigación en dichos comités.

\section{Resultados}

La primera cuestión se recoge genéricamente, en la Tabla 1.

Tabla 1- ¿Soléis jugar juntos los niños y las niñas durante el recreo y en el tiempo

\begin{tabular}{|c|c|c|c|c|c|c|c|}
\hline \multicolumn{2}{|c|}{ Colegio A } & \multicolumn{2}{|c|}{ Colegio B } & \multicolumn{2}{|c|}{ Colegio C } & \multicolumn{2}{|c|}{ ColegioD } \\
\hline & C & C & $\mathbf{C}$ & $\mathrm{C}$ & $\mathbf{C}$ & $\mathbf{C}$ & C \\
\hline hicas & hicos & hicas & hicos & hicas & hicos & hicas & hicos \\
\hline & $\Gamma$ & $\overline{\mathrm{N}}$ & $\mathrm{N}$ & $\overline{\mathrm{N}}$ & $\overline{\mathrm{N}}$ & $\overline{\mathrm{N}}$ & $\overline{\mathrm{N}}$ \\
\hline$: 0$ & $: 0$ & $: 3 \%$ & $: 3.5 \%$ & $: 8 \%$ & $: 0$ & $: 4 \%$ & $: 7 \%$ \\
\hline & $R$ & $\mathrm{R}$ & $\mathrm{R}$ & $\mathrm{R}$ & $\mathrm{R}$ & $\mathrm{R}$ & $\mathrm{R}$ \\
\hline V: 0 & $\mathrm{~V}: 0$ & $\mathrm{~V}: 0$ & $\mathrm{~V}:$ & V: $18.5 \%$ & $\mathrm{~V}:$ & $\mathrm{V}: 8 \%$ & $\mathrm{~V}: 0 \%$ \\
\hline & $A$ & A & $12,5 \%$ & A & $16.5 \%$ & A & A \\
\hline $\mathrm{V}: 32 \%$ & V & V: $46 \%$ & A & V: $39 \%$ & A & $\mathrm{V}: 48 \%$ & $\mathrm{~V}: 55 \%$ \\
\hline & $45 \%$ & A & V: $28 \%$ & A & $\mathrm{V}:$ & A & A \\
\hline M: & & A M $11 \%$ & A & $\mathrm{M}$ & $37.5 \%$ & M $24 \%$ & M: $21 \%$ \\
\hline $45 \%$ & M: & $S$ & M 9\% & $18.5 \%$ & A & $\mathrm{S}$ & S \\
\hline & $22 \%$ & $: 40 \%$ & S & S: & M: $25 \%$ & $: 16 \%$ & $: 17 \%$ \\
\hline$: 23 \%$ & $: 22 \%$ & $S$ & $: 47 \%$ & $16 \%$ & $21 \%$ & & \\
\hline
\end{tabular}

Fuente: Elaboración propia 
En el ítem 1 se destaca que en el Colegio A, un $45 \%$ de las chicas afirma que "A menudo" suelen jugar juntos niños y niñas durante el recreo y en su tiempo libre, En los chicos la opción más clara es "A veces" (45\%). De la misma manera en el Colegio C, un $46 \%$ de las chicas manifiestan que "A veces" juegan con los chicos durante el recreo y "Siempre" un 40\%. Los chicos revelan que "Siempre" (47\%) y "A veces" (28\%). En el Colegio D, un $48 \%$ de las chicas declaran que "A veces" suelen jugar juntos niños y niñas durante el recreo y el tiempo libre. El 55\% de los chicos opinan que "A veces" juegan juntos.

En general, 44,5\% de las niñas y 41,37\% de los chicos afirman que "A veces" suelen jugar juntos.

Los resultados del ítem 2 se recogen en la Tabla 2.

Tabla 2- ¿Soléis jugar a los mismos juegos los niños y las niñas durante el recreo o en vuestro tiempo libre?

\begin{tabular}{|c|c|c|c|c|c|c|c|}
\hline \multicolumn{2}{|c|}{ Colegio A } & \multicolumn{2}{|c|}{ Colegio B } & \multicolumn{2}{|c|}{ Colegio C } & \multicolumn{2}{|c|}{ ColegioD } \\
\hline & & Ch & $\mathbf{C}$ & C & C & $\mathbf{C}$ & C \\
\hline hicas & hicos & icas & hicos & hicas & hicos & hicas & hicos \\
\hline & $\mathrm{S}$ & Si: & $\mathrm{S}$ & S & $\mathrm{S}$ & $\bar{S}$ & $\bar{S}$ \\
\hline i: $73 \%$ & i: $83 \%$ & $37 \%$ & i: $47 \%$ & $\mathrm{i}: 23 \%$ & i: $54 \%$ & i: $76 \%$ & $\mathrm{i}: 45 \%$ \\
\hline & $\Lambda$ & No & $\mathrm{N}$ & 1 & $\mathrm{~N}$ & $\mathrm{~N}$ & $\mathrm{~N}$ \\
\hline o: $27 \%$ & o: $17 \%$ & $: 63 \%$ & o. $53 \%$ & $0: 77 \%$ & o: $46 \%$ & o: $34 \%$ & o: $55 \%$ \\
\hline
\end{tabular}

Fuente: Elaboración propia

En ellos se destacan que en el Colegio A, un $73 \%$ de las alumnas y el $83 \%$ de los alumnos dice que "Sí" suele jugar a los mismos juegos. En el Colegio B, 77\% de las chicas no suelen jugar a los mismos juegos. En el Colegio C, un $63 \%$ de las alumnas opinan que "No" suele jugar a los mismos juegos que los niños. En el Colegio D, un 76\% de las niñas manifiestan que "Sí" suelen jugar al mismo tipo de juegos que los niños, que consideran en un 55\% que "No", y el restante $45 \%$ opina que sus juegos son similares. 
De forma global este interrogante es contestado afirmativamente por un $69,75 \%$ de las alumnas y el $58,75 \%$ de los alumnos.

Se quiso conocer a través de la pregunta $3^{\mathrm{a}}$ el punto de vista de los alumnos y alumnas sobre si creen que hay juegos solo para niños (Tabla 3).

\begin{tabular}{|c|c|c|c|c|c|c|c|}
\hline \multicolumn{2}{|c|}{ Colegio A } & \multicolumn{2}{|c|}{ Colegio B } & \multicolumn{2}{|c|}{ Colegio C } & \multicolumn{2}{|c|}{ Colegio D } \\
\hline & & $\mathrm{C}$ & $\mathrm{C}$ & C & C & $\mathrm{C}$ & $\mathrm{C}$ \\
\hline hicas & hicos & hicas & hicos & hicas & hicos & hicas & hicos \\
\hline & $\mathrm{S}$ & $\mathrm{S}$ & $\mathrm{Si}$ & $S$ & $\mathrm{~S}$ & $\mathrm{~S}$ & $\mathrm{~S}$ \\
\hline i: $86 \%$ & i: $89 \%$ & i: $40 \%$ & $: 25 \%$ & i: $4 \%$ & i: $17 \%$ & i: $57 \%$ & i: $69 \%$ \\
\hline & & $\mathrm{N}$ & $\mathrm{N}$ & & $N$ & $\mathrm{~N}$ & $\mathrm{~N}$ \\
\hline o: $14 \%$ & o: $11 \%$ & o: $60 \%$ & o: $75 \%$ & о: $96 \%$ & o: $83 \%$ & o: $43 \%$ & o: $31 \%$ \\
\hline
\end{tabular}

Fuente: Elaboración Propia

En el Colegio A, las respuestas de ambos colectivos son similares, un $86 \%$ de alumnas y un $89 \%$ de los alumnos dicen que hay juegos solo de niños. En el Colegio B, la opinión de ambos géneros es casi unánime. En el Colegio $\mathrm{C}$, la respuesta más valorada es "No". En el Colegio D, también coinciden en sus apreciaciones, el $57 \%$ de las chicas y el $69 \%$ de los niños dice "Si".

En síntesis $74,5 \%$ de chicas y $79 \%$ de chicos niegan que haya juegos exclusivamente masculinos.

La quarta pregunta (Tabla 4) se planteó de forma diferente a los chicos y a las chicas. 
Tabla 4 - ¿Permitís que las niñas jueguen con vosotros a todos los juegos?; Y ¿Os dejan jugar los niños con ellos a todos los juegos?

\begin{tabular}{|c|c|c|c|c|c|c|c|}
\hline \multicolumn{2}{|c|}{ Colegio A } & \multicolumn{2}{|c|}{ Colegio B } & \multicolumn{2}{|c|}{ Colegio C } & \multicolumn{2}{|c|}{ Colegio D } \\
\hline & ( & & & & $\mathbf{C}$ & $\bar{C}$ & $\bar{C}$ \\
\hline hicas & hicos & icas & hicos & hicas & hicos & hicas & hicos \\
\hline & & & & 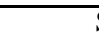 & $\mathrm{S}$ & $\mathrm{S}$ & $\bar{S}$ \\
\hline i: $77 \%$ & i: $83 \%$ & $60 \%$ & i: $56 \%$ & i: $46 \%$ & i: $100 \%$ & i: $90 \%$ & i: $31 \%$ \\
\hline & & & 1 & & $\mathrm{~N}$ & $\mathrm{~N}$ & $\mathrm{~N}$ \\
\hline o: $23 \%$ & o: $17 \%$ & $: 40 \%$ & o: $44 \%$ & o: $54 \%$ & o: $0 \%$ & o: $10 \%$ & о: $69 \%$ \\
\hline
\end{tabular}

Fuente: Elaboración Propia

Los resultados son especialmente reveladores cuando se pregunta a los encuestados sobre si los chicos permiten que las chicas jueguen en todos los juegos. Esta cuestión es preocupante, ya que en la primera parte se pregunta a los chicos si "permiten" jugar a las chicas y no se pregunta a las chicas si "dejan" jugar a los chicos, otorgando a los varones un dominio de la situación que no debería plantearse. En la segunda parte se les pregunta a las chicas si los chicos "las dejan jugar", es el mismo planteamiento realizado desde la otra perspectiva. El ítem se mantiene porque no se ha cuestionado el problema que supone plantear así los interrogantes, ni por parte de los evaluadores ni de los escolares. En este sentido, la idea se ha obtenido de la entrevista previa realizada a otros alumnos y evidencia las diferencias que siguen existiendo en la educación, en cuanto a los estereotipos de género se refiere.

$\mathrm{Si}$ articulamos las opiniones de los cuatro centros observamos que un 68,25\% de las contestaciones femeninas afirman que los niños las dejan participar en sus juegos y algo más elevado es el porcentaje de niños que dicen dejarlas jugar (74,75\%).

La percepción acerca de si los chicos permiten jugar a las chichas con ellos en todos los juegos se recoge en el ítem 5 y los resultados se aprecian en la Tabla 5. 
Tabla 5 - ¿Cuándo jugáis con las niñas las dejáis de lado? Y ¿Cuándo jugáis con los niños os sentís rechazadas?

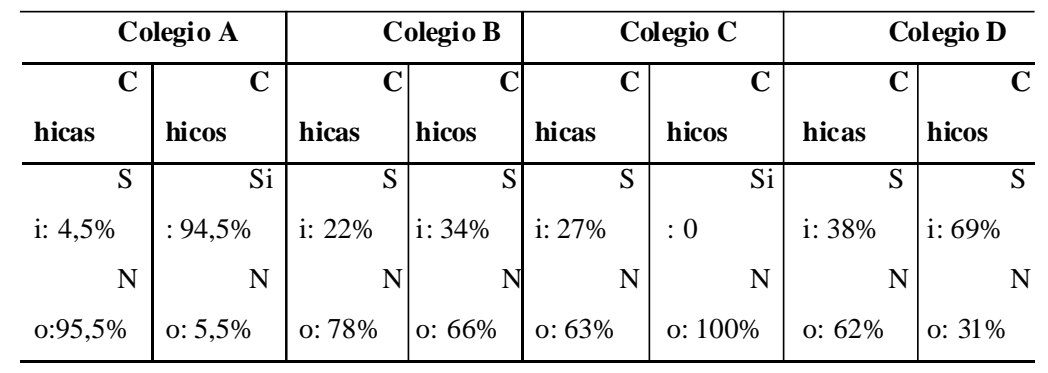

Fuente: Elaboración Propia

En el Colegio A, encontramos unas percepciones diferentes, ya que a la vista de los datos hay desacuerdo entre niños y niñas. En el Colegio B, el 27\% de las niñas declara que "Sí" se sienten rechazadas cuando juegan con los niños. En el Colegio C, el 78\% de las escolares exponen que "No" se sienten rechazadas. Y los chicos dicen que no dejan de lado a las niñas mientras juegan (66\%). Así, en el Colegio D, encontramos discrepancia en cuanto a las opiniones de chicos y chicas, el $62 \%$ de ellas manifiesta que "No" se sienten rechazadas cuando juegan con los niños mientras que un $69 \%$ de ellos confiesan que "Sí" dejan de lado a sus compañeras.

$\mathrm{Si}$ agrupamos las opiniones aparecidas en los cuatro colegios, la mayoría de las niñas $(71,5 \%)$ niegan sentirse rechazadas y un $82,25 \%$ de los chicos confirma no rehusar a las chicas.

Las percepciones y sensibilidades de los escolares sobre si cuando se eligen jugadores para participar en deportes colectivos dejan a las chicas en último lugar las recogimos con el ítem 6 (Tabla $6)$. 
Tabla 6 - Cuando hacéis equipos para jugar ¿elegís a las niñas en último lugar? y Cuando hacéis equipos para jugar, ¿los niños os eligen en último lugar?

\begin{tabular}{|c|c|c|c|c|c|c|c|}
\hline \multicolumn{2}{|c|}{ Colegio A } & \multicolumn{2}{|c|}{ Colegio B } & \multicolumn{2}{|c|}{ Colegio C } & \multicolumn{2}{|c|}{ Colegio D } \\
\hline $\mathbf{C}$ & C & $\bar{C}$ & $\bar{C}$ & $\overline{\mathbf{C}}$ & $\mathbf{C}$ & $\bar{C}$ & C \\
\hline hicas & hicos & hicas & hicos & hicas & hicos & hicas & hicos \\
\hline $\mathrm{S}$ & $\mathrm{S}$ & $\mathrm{S}$ & $\bar{S}$ & $\mathrm{~S}$ & $S$ & $\mathrm{~S}$ & $\bar{S}$ \\
\hline i: $64 \%$ & i: $17 \%$ & i: $57 \%$ & i: $69 \%$ & i: $54 \%$ & i $21 \%$ & i: $5 \%$ & i: $52 \%$ \\
\hline $\mathrm{N}$ & $N$ & $\mathrm{~N}$ & $\mathrm{~N}$ & $\mathrm{~N}$ & $\mathrm{~N}$ & $\mathrm{~N}$ & $\mathrm{~N}$ \\
\hline o: $46 \%$ & o: $83 \%$ & o: $43 \%$ & o: $31 \%$ & o: $46 \%$ & o: $79 \%$ & o: $95 \%$ & o: $48 \%$ \\
\hline
\end{tabular}

Fuente: Elaboración Propia

Se destaca que en el Colegio A, los datos muestran que las chicas afirman (64\%) que los niños "Sí" las eligen en último lugar cuando hacen equipos, sin embargo, la mayoría de los chicos opinan de forma contraria, el $83 \%$ considera que "No" las dejan de lado a la hora de elegir compañeros de equipo. En el Colegio $\mathrm{C}$, los chicos declaran que "Si", eligen a las chicas en último lugar para conformar un equipo a la hora de jugar (69\%). Por último en el Colegio D, las opiniones son dispares, el $95 \%$ del colectivo femenino considera que los niños no las eligen en último lugar, mientras que en el masculino las opiniones están más igualadas ya que el $52 \%$ se posicionan en la opción "Sí".

Congregadas todas las opiniones, prácticamente la mitad $(56,75 \%)$ de las alumnas, opina que son elegidas en último lugar por los chicos. Ellos dicen no escogerlas en último lugar en un $72 \%$ de los casos.

A menudo en nuestra sociedad, se atribuye a los hombres mejor condición física que a las mujeres. Dado el importante peso que sigue teniendo aún la atribución de debilidad a la mujer como rasgo característico respecto al hombre formulamos el doble ítem 7 para chicos y para chicas (Tabla 7). 
Tabla 7- ¿Creéis que las niñas son más débiles y no pueden hacer algunos deportes que requieren más esfuerzo físico? y ¿Los niños creen que sois más débiles y no os dejan participar en actividades que requieren mucho esfuerzo?

\begin{tabular}{|c|c|c|c|c|c|c|c|}
\hline \multicolumn{2}{|c|}{ Colegio A } & \multicolumn{2}{|c|}{ Colegio B } & \multicolumn{2}{|c|}{ Colegio C } & \multicolumn{2}{|c|}{ Colegio D } \\
\hline $\mathbf{C}$ & & C & $\mathbf{C}$ & $\mathbf{C}$ & $\mathbf{C}$ & $\mathbf{C}$ & C \\
\hline hicas & hicos & hicas & hicos & hicas & hicos & hicas & hicos \\
\hline $\mathrm{S}$ & $\mathrm{S}$ & $\mathrm{S}$ & $\mathrm{S}$ & $\mathrm{S}$ & $\mathrm{S}$ & $\mathrm{S}$ & $\mathrm{S}$ \\
\hline i: $14 \%$ & i: $38 \%$ & i: $51 \%$ & i: $50 \%$ & i: $54 \%$ & i: $42 \%$ & i: $14 \%$ & i: $38 \%$ \\
\hline $\mathrm{N}$ & $\mathrm{N}$ & $\mathrm{N}$ & $\mathrm{N}$ & $\mathrm{N}$ & $\mathrm{N}$ & $\mathrm{N}$ & $\mathrm{N}$ \\
\hline o: $86 \%$ & o: $72 \%$ & o: $49 \%$ & o: $50 \%$ & o: $12 \%$ & o: $58 \%$ & o: $86 \%$ & o: $62 \%$ \\
\hline
\end{tabular}

Fuente: Elaboración Propia

Podemos ver que en el Colegio B, las opiniones están muy igualadas, un 54\% opina que "Sí", que los niños no las dejan participar en deportes o actividades que requieren mucho esfuerzo, mientras que el $46 \%$ restante declara que "No", que pueden jugar junto a los chicos y no son juzgadas como débiles para ninguna actividad deportiva. En el Colegio D, los datos revelan una aproximación en las opiniones, el "No" es la opción más elegida, $86 \%$ de ellas y $62 \%$ de ellos. Ambos manifiestan que las niñas no son más débiles y están capacitadas para practicar cualquier deporte o AF.

De manera genérica un $67,25 \%$ de las chicas no cree que los niños piensen que son más débiles y un $61,5 \%$ de los niños tampoco cree que ellas lo sean.

Para conocer en qué medida se enfadan las niñas cuando los chicos no las dejan jugar y si los chicos percibían dichos enojos, planteamos el ítem 8 (Tabla 8). 
Tabla 8 - ¿Os fijáis si las niñas se enfadan cuando cuándo las dejáis de lado ó las elegís en último lugar para jugar a algún deporte? y ¿Os enfadáis cuando los niños os rechazan y no os eligen para jugar a algún deporte?

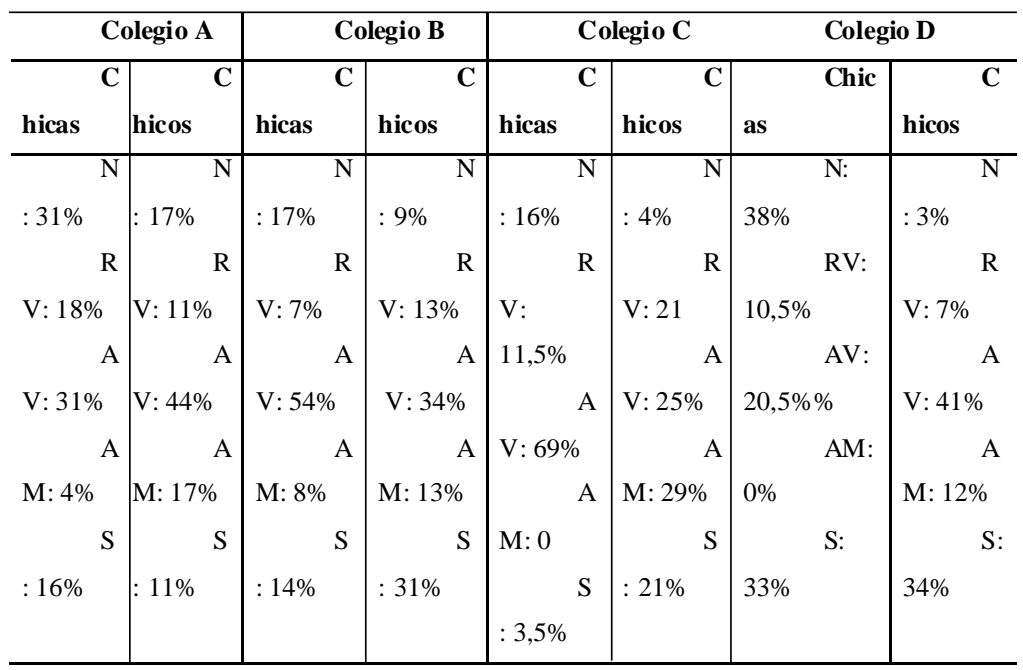

Fuente: Elaboración Propia

En el Colegio A, un 32\% de las niñas manifiestan que "Nunca". Los chicos descubren que "A veces" (44\%), se percatan del enfado de sus compañeras. En el Colegio B, el $69 \%$ las chicas considera que "A veces" se enfadan cuando los niños las rechazan en un juego, mientras que los chicos "A menudo" (29\%) advierten que ellas se enojan cuando las rechazan para jugar. En el Colegio C, el 54\% de ellas dice que "A veces" se enfadan cuando sus compañeros las rechazan. En el Colegio D, la opción más secundada entre las niñas es "Nunca" (38\%), seguida de "Siempre" (33\%). En el caso de los niños, la respuesta más repetida es "A veces" (41\%).

Integrando los resultados, un $24,25 \%$ de las chicas dice no enfadarse "Nunca" cuando no son elegidas para jugar por sus compañeros. Los chicos en un $36 \%$ de los casos son conscientes "A veces" de que las chicas se sienten molestas al no ser escogidas para jugar. 
La discriminación suele tener una connotación negativa en la medida en que se trata despectivamente o se perjudica a un determinado grupo. En el ítem 9 se incide en dicha cuestión (Tabla 9).

Tabla 9 - ¿Te ríes de las niñas cuando pierden en un juego? y ¿Los niños se ríen de ti cuando pierdes?

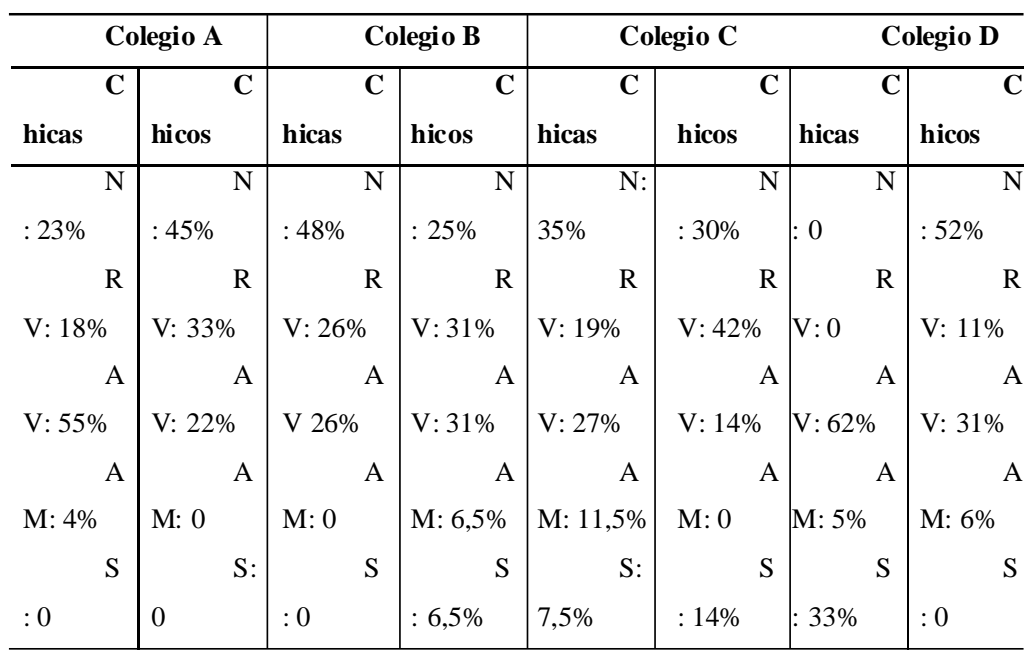

Fuente: Elaboración Propia

En el Colegio A, las alumnas exponen "A veces" (55\%) los niños se ríen de ellas cuando pierden, la opción "Siempre" no ha sido elegida en ningún caso. Para los chicos, la respuesta que más aparece es "Nunca" (45\%), las respuestas "A menudo" y "Siempre" no han sido consideración entre los chicos. En el Colegio B, la respuesta mayoritaria entre las chicas ha sido "Nunca" (35\%). Entre los chicos la opción más considerada es "Rara Vez" (42\%). En el Colegio D, el $62 \%$ de las niñas considera que "A veces" los niños se ríen de ellas cuando pierden. El 52\% de los niños expone que "Nunca" se ríe de sus compañeras.

De manera global podemos observar que "A veces" es la respuesta que más se repite $(65,25 \%)$ por parte de las niñas. Los chicos, afirman no reírse "Nunca" de ellas cuando pierden en algún juego $(31,25 \%)$. 
Finalmente el ítem 10 (Tabla 10) pretende conocer la percepción acerca de si chicos y chicas prefieren participar en juegos y deportes juntos o por el contrario, separados.

Tabla 10 - Cuando tienes que elegir un compañero o formar un equipo, ¿prefieres que todos seáis chicos? y para las niñas, ¿prefieres que todas seáis chicas?

\begin{tabular}{|c|c|c|c|c|c|c|c|}
\hline \multicolumn{2}{|c|}{ Colegio A } & \multicolumn{2}{|c|}{ Col egio B } & \multicolumn{2}{|c|}{ Colegio C } & \multicolumn{2}{|c|}{ Colegio D } \\
\hline $\mathbf{C}$ & C & $\mathrm{Ch}$ & Q & $\mathbf{C}$ & $\mathbf{C}$ & $\mathbf{C}$ & $\mathbf{C}$ \\
\hline hicas & hicos & icas & hicos & hicas & hicos & hicas & hicos \\
\hline $\bar{S}$ & $\mathrm{~S}$ & Si: & $\mathrm{S}$ & $\bar{S}$ & $\mathrm{~S}$ & $\mathrm{~S}$ & $\mathrm{~S}$ \\
\hline i: $27 \%$ & i: $17 \%$ & $31 \%$ & i: $19 \%$ & i: $23 \%$ & i: $17 \%$ & i: $62 \%$ & i: $52 \%$ \\
\hline $\mathrm{N}$ & $\mathrm{N}$ & $\mathrm{No}$ & $N$ & $\mathrm{~N}$ & $\mathrm{~N}$ & $\mathrm{~N}$ & $\mathrm{~N}$ \\
\hline o: $73 \%$ & o: $83 \%$ & $: 69 \%$ & o: $81 \%$ & o: $77 \%$ & o: $83 \%$ & o: $28 \%$ & o: $48 \%$ \\
\hline
\end{tabular}

Fuente: Elaboración Propia

En el Colegio A, ambos géneros coinciden en sus respuestas, siendo "No" la opción mayoritaria $(73 \%)$ entre ellas y $83 \%$ entre ellos. En el Colegio B, un $77 \%$ de las chicas declara que no prefiere que los equipos estén formados únicamente por chicas. $83 \%$ de los chicos opina lo mismo. En el Colegio D, un 62\% de las niñas "Sí" prefiere que los equipos sean formados íntegramente por chicas, mientras que los chicos, solo el $52 \%$ prefiere equipos exclusivamente masculinos. En total, un 66,75\% de las niñas y el 73,75\% de los chicos prefieren formar equipos mixtos para jugar.

\section{Discusión}

A la vista de los resultados, se observa que el concepto de discriminación por sexo está todavía presente en el ámbito del juego escolar y deportivo, aunque las respuestas varíen de un colegio a otro. Los datos obtenidos no demuestran si esta tendencia comienza a desaparecer entre los escolares que van asimilando el concepto de igualdad. Los resultados apuntan a que se está produciendo un cambio de conducta, ya que niños y niñas cada vez comparten más aficiones, lo que les permite realizar AF conjuntamente. 
Bien es cierto que cada sociedad establece pautas, reglas y leyes que determinan lo que debe y no debe hacerse; pero debido a que cada cultura define de diferente manera sus normas, y en consecuencia lo que es una trasgresión y lo que no, las conductas consideradas "divergentes" varían de lugar a lugar y de tiempo en tiempo.

A modo de crítica de nuestro trabajo, señalamos lo ya argumentado en el ítem 4 sobre haber mantenido la palabra "permitir" para preguntar a los chicos si dejan a las chicas jugar y viceversa. Creemos que, desde la perspectiva de igualdad de género en un contexto educativo, esa palabra debería de estar erradicada en lo que a compartir actividades se refiere.

Las alumnas defienden que ellas no tienen que negociar y desafiar formas sexistas y racistas para, simplemente, ser más activas físicamente. En este sentido, coincidimos con los resultados hallados en la investigación de Valdivia et al.1 (in press), y así, pese a percibirse un estereotipo más igualitario entre los niños, sí que en determinados colegios, con una población escolar circunstancial, se han hallado resultados más relacionados con la realidad descrita en Oliver y Hamzeh (2010), es decir, que las respuestas de niños y niñas en el presente estudio se han equilibrado, pero nunca llegando a igualarse. Con dicho planteamiento se coincide con el estudio de Curnow y McDonald (1995) en el que se afirma que en algunos contextos los chicos han llegado a ridiculizar los esfuerzos de las chicas y la mayoría de las veces son ellos los que asumen roles de poder como capitanes de los equipos.

Se advierte que las formas de jugar y hacer deporte están muy interrelacionadas con las condiciones del medio sociocultural del contexto (NEUBER, 2002). Observamos, como señalan Van Acker, Carreiro da Costa y Bourdeaudhuij (2010), que los juegos de invasión se pueden utilizar como instrumento para aumentar los niveles de AF en ambos sexos. Dichas actividades también son resaltadas, en el mismo sentido, en el estudio sobre género de Valdivia et al. (2012).

En definitiva, dado que tanto chicos como chicas están muy 
interesados en la igualdad por encima de todo, cuestión que coincide también con el trabajo de Oliver, Hamzeh y McCaunghtry (2009), se precisa un modelo curricular de intervención educativa que sea efectivo, comprenda las características de los niveles de habilidad de chicos y chicas, y tenga en cuenta las cualidades de todos los alumnos por igual, ambos aspectos que deben darse dentro de la EF en los colegios.

Todo ello, como indican Dyson, Linehan y Hastie (2010), a través de una interdependencia positiva entre los escolares que se produce cuando perciben que están unidos a miembros del grupo, de tal manera que no pueden tener éxito sin los otros. Las chicas, además, consideran que existe cierta crisis de igualdad en las oportunidades de participación en AF. Tienen ideas de cómo cambiar las desigualdades, pero lo que no tienen es un lugar en la escuela o en el currículo, para desafiarlas.

\section{Conclusiones}

Existe algún hecho aislado de discriminación en función del género, pero en las ocasiones donde niños y niñas juegan separados es porque ellos y ellas lo prefieren así. No obstante, en estas edades (11-12 años) los niños y las niñas tienden a juntarse en grupos homogéneos, en cuanto al género se refiere. De esta manera se pone de manifiesto un aprendizaje cultural.

Más de la mitad de niños y niñas practican los mismos juegos en su tiempo de ocio, por lo que las diferencias entre unos y otras es cada vez menor.

El 75\% de los niños reconocen "permitir" jugar con ellos a las niñas $y$, en un porcentaje menor pero próximo, las niñas afirman que los chicos las dejan jugar, aunque consideran que son rechazadas a la hora de conformar equipos para juegos colectivos, suceso que no es percibido por los chicos.

Un bajo porcentaje piensa que hay juegos y deportes exclusivos de chicos, pero cuando ambos colectivos lo desean, niños y niñas 


\section{ArtigosOriginais}

participan de manera conjunta, salvo casos aislados de niñas que dicen sentirse rechazadas, circunstancia que los chicos niegan.

Muchas niñas creen que son elegidas en último lugar por los chicos cuando se forman los equipos. Los chicos, en un alto porcentaje, opinan lo contrario y además afirman no reírse de las chicas cuando pierden. 


\begin{abstract}
As meninas também querem brincar: a participaçao conjunta de meninos e meninas em atividades físicas näo organizadas no contexto escolar.

Resumo: Apresentamos um estudo empírico descritivo realizado com 250 participantes do sexto ano da Educação Primária (EP), 130 meninas e 120 meninos de quatro centros escolares espanhóis com diferentes características, aplicando-se uma enquete de 10 itens para compreender os obstáculos que encontram as meninas na realização de atividade física (AF) e esporte no tempo de lazer escolar. Os resultados indicam que existem diferenças de gênero na hora de praticar jogos e esportes, ainda que se apresentem mudanças de conduta que fazem que meninos e meninas se agruparem com maior frequência para realizar atividades físicas e esportes.
\end{abstract}

Palavras-chave: Atividade motora. Esportes. Atividades de lazer. Identidade de gênero. Ensino fundamental e médio.

Also want girls play: participation of children in organized physical activity in the context no school.

Abstract: We present a descriptive empirical study carried out with 250 pupils belonging to 6th of Primary Education (EP), 130 girls and 120 boys, from four schools with different characteristics to which there is applied a survey of 10 questions with the aim of understanding the barriers that girls find when they are accomplishing physical activities (AF), during their leisure time out of school. The results show the difference of gender exists when practising games and sport, though a change of conduct is taking place, since children and girls every time share more the accomplishment of physical and sports activities. The ways of playing and playing sports, are very interrelated with socio-cultural environment.

Keywords: Motor activity. Sports. Leisure activities. Gender identity. Education. primary and secondary. 


\section{REFERÊNCIAS}

AZZARITO, L.; SOLMON, M.A. A post-structural analysis of high school's gendered and racialized bodily meanings. Journal of Teaching in Physical Education, Champaing, v. 25, p. 75-98, 2006.

BIDDLE, S.J.H.; GORELY, T.; STENSEL, D.J. Health-Enhancing Physical Activity and Sedentary Behavior in Children and Adolescents. Journal of Sports Science, Oxford, v. 22, p. 679-701, 2004.

CASTILLO, M.A.; MARTÍNEZ-LÓPEZ, E.J. ; ZAGALAZ, M.L. Analysis of the physical education teacher's opinion from the Lakes Region (Chile) about the classes based on a mixed modality. Journal of Sport and Health Research, Jaén, v. 2, n. 2, p. 77-94, 2010.

CERVELLÓ, E.; JIMÉNEZ, R.; DEL-VILLAR, F.; RAMOS, L.; SANTOS-ROSA, F.J. Goal orientations, motivational climate, equality and discipline of Spanish Physical Education students. Perceptual and Motor Skills, Missoula, v. 99, p. 271-283, 2004.

CURNOW, J.; MCDONALD, D. Can Sport Education be gender inclusive: a case study in an upper Primary School. Australian Council for Health. ACHPER Healthy Lifestyles Journal, Hindmarsh, v. 42, p. 9-11, 1995.

DWYER, J.J.M.; ALLISON, K.R.; GOLDENBERG, E.R.; FEIN, A.J.; YOSHIDA, K.K. ; BOUTILIER, M.A. Adolescent Gils Perceived Barriers to Participation in Physical Activity. Adolescence, San Diego, v. 41, p. 75-89, 2006.

DYSON, B.P.; LINEHAN, N.R.; HASTIE, P.A. The Ecology of Coopertive Learning in Elementary Physical Education Classes. Journal of Teaching in Physical Education, Champaign, v. 29, p. 113-130, 2010.

EISNMANN, J.C.; KATZNARYK, P.T. ; TREMBLAY, M.S. Leisure-time physical activity levels among Canadian adolescents, 1981-1998. Journal of Physical Activity and Health, Champaign, v. 1, p. 154-162, 2004.

EUROPEAN COMMITTE FOR SOCIAL COHESION. Revised strategy for social cohesion. Estrasburgo: Council of Europe, 2004.

FLINTOFF, A. Y SCRATON, S. Stepping into Active Leisure? Young Women's Perceptions of Active Lifestyles and their Experiences of School Physical Education. Sport Education and Society, Oxford, v 6, n. 1, p. 5-22, 2001.

GIL-MADRONA, P. ; CONTRERAS-JORDÁN, O. Interés y valoración del área de EF por padres y alumnos en la enseñanza obligatoria. Revista de Educación, Madrid, v. 332, p. $255-283,2003$.

$\mathrm{HAMZEH}, \mathrm{M}$. A de-veiling narrative inquiry: Entry and agency in body stories of Muslim girls. Dissertation (Doctoral). New Mexico State University. 2007. 
INCLUD-ED. Strategies for inclusion and social cohesion from education in Europe. Bruselas, 2011.

INSTITUTO DE LA MUJER. Estudio sobre actitudes y prácticas deportivas de las mujeres españolas: 1990-2005. Madrid, 2005.

JANSSEN, I.; KATZMARZYK, P.T.; BOYCE, W.G. ; PICKETT, W. The independent influence of physical inactivity and obesity on health complaints in 6th and 10th grade Canadian youth. Journal of Physical Activity and Health, Champaign, v. 1, p. 331-343, 2004.

JIMÉNEZ, R.; GARCÍA, T.; SANTOS-ROSA, F. J.; MORENO, A. ; CERVELLÓ, E. Análisis de las relaciones entre orientaciones de meta, clima motivacional, valoración de la Educación Física y flow disposicional en estudiantes de Educación física en Secundaria. Infancia y Aprendizaje, Madrid, v. 33, n. 1, p. 107-116, 2010.

NEUBER, N. (2002). Entwicklungsforderung durch Bewegung?-Methodologiche Überlegungen zu einer sportpädagogischen Jugendforschung. En: G. FRIEDRICH (Eds.), Sportpädagogische ForsChung. Konzepte, Projeskte, Perspektiven Schriften der Deutschen Vereinigung für Sportwissenschaft. Hamburg, Czwalina,, 2002. p. 300-206.

OLIVER, K. L.; HAMZEH, M., ; MCCAUGHTRY, N. Grily girls can play games. Cocreating a curriculum of possibilities with 5th grade grils. Journal of Teaching in Physical Education, Champaign, v. 28, p. 90-110, 2009.

OLIVER, K.L. ; HAMZEH, M. The boys won't Let Us Play: Fitth-Grade Mestizas Challenge Physical Activity Discourse at School. Researh Quarterly for Exercise and Sport, Reston, v. 81, n. 1, p. 38-51, 2010.

ORTEGA, E.; CALDERÓN, A.; PALAO, J. M. ; PUIGCERVER, C. Diseño y validación de un cuestionario para evaluar la actitud percibida del profesor en clase y un cuestionario para evaluar los contenidos actitudinales de los alumnos durante las clases de Educación Física en secundaria. Retos: Nuevas tendencias en Educación Física, Deportes y Recreación, Murcia, v. 14, p. 22-29, 2008.

RIDDOCH, C.J.; ANDERSON, L.B.; WEDDERKOPP, N.; HARRO, M.; KLASSONHEGGBO, L. Y SARDINHA, L.B. Physical activity levels and patterns of 9 and 15 year-old European children. Medicine \& Science in Sport \& Exercise, Indianapolis, v. 36, p. 86-92, 2004.

VALDIVIA-MORAL, P.; LÓPEZ-LÓPEZ, M.; LARA-SÁNCHEZ, A. Y ZAGALAZSÁNCHEZ, M.L. Concepto de coeducación en el profesorado de EF y metodología utilizada para su trabajo. Movimento, Porto Alegre, v. 18, n. 4, p. 197-217, 2012.

VAN ACKER, R.; CARREIRO DA COSTA, F..; BOURDEUDHUIJ DE, I. Sex equity and physical activity levels in coeducational physical education: exploring the potential of modified game forms. Physical Education and Sport Pedagogy, Oxford, v. 15, n 2, p. 159-173, 2010. 


\section{ArtigosOriginais}

WILSON, D.K.; WILLIAMS, J.; EVANS, A.; MIXON, G. ; RHEAUME, C. Brief report: A qualitative study of gender preferences and motivational factors for physical activity in underserved adolescents. Journal of Pediatric Psychology, Oxford, v. 30, p. 293-297, 2005.

WOODHILL, B.M. ; SAMUELS, C.A. Desirable and Undesitable Androgyny: A Prescription for the Twenty-First Century. Journal of Gender Studies, Oxford, v. 13, n. 1, p. 15-28, 2004.

ZAGALAZ-SÁNCHEZ, M.L.; PANTOJA-VALLEJO, A.; MARTÍNEZ-LÓPEZ, E.J.; ROMERO-GRANADOS, S. La EF escolar desde el punto de vista del alumnado de educación primaria y del estudiante de magisterio. Revista de Investigación

Educativa, Murcia, v. 26, n. 2, p. 347-369, 2008.

Endereço para correspondência:

Dr. Pedro Gil Madrona

Universidad de Castilla la Mancha.

Facultad de Educación, Campus Universitario de Albacete.

C.P. 02071, Albacete, España.

Recebido em: 22. 03. 2013

Aprovado em: 02.10.2013 